\title{
Stability aspects of arithmetic functions
}

\author{
by \\ Tomasz Kochanek (Katowice)
}

1. Introduction. In number theory a significant role is played by two classes of arithmetic mappings: the so called arithmetic additive functions and arithmetic multiplicative functions.

Definition 1. A function $f: \mathbb{N} \rightarrow \mathbb{C}$ is called arithmetic additive if

$$
f(x y)=f(x)+f(y)
$$

for all $x, y \in \mathbb{N}$ such that $(x, y)=1$. It is called arithmetic multiplicative if $f \neq 0$ and

$$
f(x y)=f(x) f(y)
$$

for all $x, y \in \mathbb{N}$ such that $(x, y)=1$.

Definition 2. In the case where equation (1.1) (or (1.2)) holds for all $x, y \in \mathbb{N}$, the function $f$ is called completely additive (respectively: completely multiplicative).

A natural stability question for arithmetic additive functions looks like this: assume that for a fixed $\varepsilon \geq 0$ we have the conditional inequality

$$
x, y \in \mathbb{N},(x, y)=1 \Rightarrow|f(x y)-f(x)-f(y)| \leq \varepsilon .
$$

Does it imply that $f$ is approximately equal to some arithmetic additive function?

Our aim is to establish two theorems which give an answer to this question under two different additional assumptions.

The first problem concerning the stability of additive mappings was raised by S. M. Ulam [13] and was partially solved by D. H. Hyers [8] in 1941. The condition " $(x, y)=1$ " appearing in (1.3) causes that the Hyers sequence method cannot be used in our case. Stability of conditional Cauchy functional equations was investigated by several authors (see, e.g., Z. Gajda [2],

2000 Mathematics Subject Classification: Primary 11K65, 39B82.

Key words and phrases: arithmetic functions, additive functions, stability, conditional functional equation, Ramsey theorem. 
R. Ger [3], R. Ger and J. Sikorska [6]). These papers concern the situation where the Cauchy equation is approximately satisfied for "almost all" pairs of arguments or for arguments orthogonal in some sense. The expression "almost all" is understood in an axiomatic way and is connected with the notion of proper linearly invariant ideal. However, the set $\left\{(x, y) \in \mathbb{N}^{2}:(x, y)=1\right\}$ neither generates any proper linearly invariant ideal, nor is a subset of any orthogonality relation. Consequently, such approaches are not applicable in the study of hypothesis (1.3).

2. Stability type results for arithmetic additive functions. Let $\mathbb{P}$ and $\mathbb{S}$ denote the sets of all prime numbers and of all natural powers of primes, respectively (i.e. $\mathbb{S}=\left\{p^{k}: p \in \mathbb{P}, k \in \mathbb{N}\right\}$ ). Let $\omega: \mathbb{N} \rightarrow \mathbb{N} \cup\{0\}$ be the standard arithmetic additive function given by

$$
\omega(x)=\operatorname{card}\{p \in \mathbb{P}: p \mid x\} \quad \text { for } x \in \mathbb{N} .
$$

We start with a result guaranteeing that, under a certain regularity assumption, an almost arithmetic additive function may be approximated by a logarithmic function. Several mathematicians were looking for conditions which force an arithmetic additive function to be of the form $c \log n$. The first two results of this type, due to P. Erdôs [1], assert that it is the case if $f$ satisfies one of the following conditions:

(i) $f$ is monotone,

(ii) $\lim _{x \rightarrow \infty}(f(x+1)-f(x))=0$.

A. Rényi in [12] gave a simplified and elegant proof of the Erdôs theorem in case (ii). Later, I. Kátai [9] and A. Máté [11] strengthened the assertion, assuming only that

(iii) $\liminf _{x \rightarrow \infty}(f(x+1)-f(x)) \geq 0$, instead of (ii) (as anticipated by Erdős). The following proposition is a natural generalization of the theorem of Erdôs-Kátai-Máté from the stability point of view.

TheOrem 1. Assume that a function $f: \mathbb{N} \rightarrow \mathbb{R}$ satisfies (1.3) and

$$
\liminf _{x \rightarrow \infty}(f(x+1)-f(x)) \geq 0 .
$$

Then there exists $c \in \mathbb{R}$ such that

$$
|f(x)-c \log x| \leq \varepsilon \quad \text { for } x \in \mathbb{N} .
$$

Proof. The first step of the proof is a slightly modified computation appearing in A. Máté's proof [11]. For $\mu>0$ define

$$
H(\mu):=\{x \in \mathbb{N}: f(x+1)-f(x)<-\mu\} ;
$$


obviously it is a finite set. Thus,

$$
c(\mu):=-\sum_{x \in H(\mu)}(f(x+1)-f(x))
$$

is a well defined, nonnegative number. Lemma 1 from [11] says that for every finite set $M \subset \mathbb{N}$ we have

$$
\sum_{x \in M}(f(x+1)-f(x)) \geq-(\operatorname{card} M) \mu-c(\mu) \quad \text { for } \mu>0 .
$$

For arbitrary $x, k \in \mathbb{N}$ we have

$$
\begin{aligned}
f\left(x^{k}\right)-k f(x)= & \left(f\left(x^{k}\right)-f\left(x^{k}-1\right)\right)+\sum_{i=2}^{k}\left(f\left(x^{i}-1\right)-f\left(x^{i-1}-1\right)-f(x)\right) \\
& +(f(x-1)-f(x)) \\
= & \left(f\left(x^{k}\right)-f\left(x^{k}-1\right)\right)+\sum_{i=2}^{k}\left(f\left(x^{i}-1\right)-f\left(x^{i}-x\right)+\varepsilon_{i}\right) \\
& +(f(x-1)-f(x)),
\end{aligned}
$$

with some $\varepsilon_{i} \in[-\varepsilon, \varepsilon]$. This follows from (1.3) and the fact that $x^{i-1}-1$ and $x$ are relatively prime for $i=2, \ldots, k$. Continuing the above calculation with the aid of (2.3), we obtain

$$
\begin{aligned}
f\left(x^{k}\right)-k f(x)= & \left(f\left(x^{k}\right)-f\left(x^{k}-1\right)\right) \\
& +\sum_{i=2}^{k} \sum_{j=0}^{x-2}\left(f\left(x^{i}-x+j+1\right)-f\left(x^{i}-x+j\right)\right) \\
& +\sum_{i=2}^{k} \varepsilon_{i}+(f(x-1)-f(x)) \\
\geq & -(1+(k-1)(x-1)) \mu-c(\mu) \\
& -(k-1) \varepsilon+(f(x-1)-f(x)) \\
\geq & -k x \mu-c(\mu)-(k-1) \varepsilon+(f(x-1)-f(x)) .
\end{aligned}
$$

Similarly,

$$
\begin{aligned}
f\left(x^{k}\right)-k f(x)= & \left(f\left(x^{k}\right)-f\left(x^{k}+1\right)\right)+\sum_{i=2}^{k}\left(f\left(x^{i}+1\right)-f\left(x^{i-1}+1\right)-f(x)\right) \\
& +(f(x+1)-f(x))
\end{aligned}
$$

and arguing as above, we deduce

$$
f\left(x^{k}\right)-k f(x) \leq k x \mu+c(\mu)+(k-1) \varepsilon+(f(x+1)-f(x)) .
$$

As a result,

$$
\left|f\left(x^{k}\right)-k f(x)\right| \leq k x \mu+c(\mu)+(k-1) \varepsilon+o(k) .
$$


The arbitrariness of $\mu$ allows us to deduce the inequality

$$
\limsup _{k \rightarrow \infty}\left|\frac{f\left(x^{k}\right)}{k}-f(x)\right| \leq \varepsilon \quad \text { for } x \in \mathbb{N} .
$$

This estimate gives us the possibility of defining a function $g(x)$ as some kind of limit (lim sup or Banach limit) of the expression $f\left(x^{k}\right) / k$. Assumption (1.3) easily implies that such a mapping $g$ is arithmetic additive; however, at present, we are not able to show that it is completely additive. To overcome this difficulty we proceed as follows.

Fix $p \in \mathbb{P}$. Our present purpose is to establish that

$$
Z_{p}:=\left\{z \in \mathbb{R}:\left|f\left(p^{\alpha}\right)-\alpha z\right| \leq \varepsilon \text { for all } \alpha \in \mathbb{N}\right\} \neq \emptyset .
$$

Observe that

$$
Z_{p}=\bigcap_{\alpha \in \mathbb{N}}\left\{z \in[f(p)-\varepsilon, f(p)+\varepsilon]:\left|f\left(p^{\alpha}\right)-\alpha z\right| \leq \varepsilon\right\} ;
$$

thus, it is an intersection of compact sets. To show that they form a centered family it is enough to prove that for any finite number $s$ of natural numbers $\alpha_{1}, \ldots, \alpha_{s}$ there exists $z \in \mathbb{R}$ such that

$$
\left|f\left(p^{\alpha_{i}}\right)-\alpha_{i} z\right| \leq \varepsilon \quad \text { for } i=1, \ldots, s .
$$

Let $\alpha:=\alpha_{1} \cdots \alpha_{s}$. In the light of (2.4), the sequence

$$
\left(\frac{f\left(x^{\alpha k}\right)}{\alpha k}\right)_{k=1}^{\infty}
$$

is bounded for every $x \in \mathbb{N}$. Choose a subsequence $k_{1}<k_{2}<\cdots$ of natural numbers such that the limit

$$
z:=\lim _{n \rightarrow \infty} \frac{f\left(p^{\alpha k_{n}}\right)}{\alpha k_{n}}
$$

exists. For every $i=1, \ldots, s$ we have, in view of $(2.4)$,

$$
\left|f\left(p^{\alpha_{i}}\right)-\alpha_{i} z\right|=\left|f\left(p^{\alpha_{i}}\right)-\lim _{n \rightarrow \infty} \frac{f\left(\left(p^{\alpha_{i}}\right)^{\left(\alpha / \alpha_{i}\right) k_{n}}\right)}{\left(\alpha / \alpha_{i}\right) k_{n}}\right| \leq \varepsilon,
$$

as desired.

By virtue of (2.5), for every $p \in \mathbb{P}$ we can select a value $z(p)$ such that $\left|f\left(p^{\alpha}\right)-\alpha z(p)\right| \leq \varepsilon, \alpha \in \mathbb{N}$. Hence,

$$
\left|\frac{f\left(p^{\alpha k}\right)}{k}-\alpha z(p)\right| \leq \frac{\varepsilon}{k} \quad \text { for } p \in \mathbb{P}, \alpha, k \in \mathbb{N},
$$

which yields

$$
\alpha z(p)=\lim _{k \rightarrow \infty} \frac{f\left(p^{\alpha k}\right)}{k} \quad \text { for } p \in \mathbb{P}, \alpha \in \mathbb{N} .
$$


Assumption (1.3) and formula (2.6) imply that if $p, q \in \mathbb{P}, p \neq q, \alpha, \beta \in \mathbb{N}$, then

$$
\alpha z(p)+\beta z(q)=\lim _{k \rightarrow \infty} \frac{f\left(p^{\alpha k}\right)+f\left(q^{\beta k}\right)}{k}=\lim _{k \rightarrow \infty} \frac{f\left(\left(p^{\alpha} q^{\beta}\right)^{k}\right)}{k},
$$

and further, by an easy induction,

$$
\sum_{i=1}^{s} \alpha_{i} z\left(p_{i}\right)=\lim _{k \rightarrow \infty} \frac{f\left(\left(p_{1}^{\alpha_{1}} \cdots p_{s}^{\alpha_{s}}\right)^{k}\right)}{k},
$$

for all mutually different $p_{1}, \ldots, p_{s} \in \mathbb{P}$ and $\alpha_{1}, \ldots, \alpha_{s} \in \mathbb{N}$. In particular, (2.7) shows that the limit

$$
\lim _{k \rightarrow \infty} \frac{f\left(x^{k}\right)}{k}
$$

exists for every $x \in \mathbb{N}$, and moreover it is equal to $g(x)$, where $g: \mathbb{N} \rightarrow \mathbb{R}$ is the unique completely additive function satisfying $g(p)=z(p)$ for $p \in \mathbb{P}$. Inequality (2.4) yields $|f(x)-g(x)| \leq \varepsilon$ for all $x \in \mathbb{N}$. Hence, to finish the proof of (2.2), it remains to prove that $g$ satisfies some condition guaranteeing that an additive function is a logarithmic function. By assumption (2.1), $f(x+1)-f(x)$ is bounded from below, hence so is $g(x+1)-g(x)$, as $g$ approximates $f$ to within $\varepsilon$. Since $g$ is completely additive, the theorem of E. Wirsing (see [14]) forces it to be of the form $g(x)=c \log x$.

In the proof of the next result we use the celebrated Ramsey theorem quoted below.

Theorem (Ramsey). Let $X$ be an infinite set and $n \in \mathbb{N}$. If the family $X[n]$ of all $n$-element subsets of $X$ is partitioned into finitely many parts:

$$
X[n]=X_{1} \cup \cdots \cup X_{k},
$$

then there exists an infinite set $Y \subset X$ such that all its $n$-element subsets lie in one part of the partition.

Assumption (2.8) appearing below states that some special expressions, like those appearing in (1.3), may be arbitrarily small. More precisely, if $\omega(r)=1$, then the difference $f(r x)-f(x)-f(r)$ tends to zero as $\omega(x)$ tends to infinity, $x$ and $r$ being coprime. Moreover, we require this convergence to be uniform with respect to $r \in \mathbb{S}$. This assumption may be regarded as quite natural, compared to the one considered by P. Erdôs, namely, assumption (ii), which can be written equivalently as $\lim _{x \rightarrow \infty}(f(x+1)-f(x)-f(1))=0$.

Theorem 2. Assume that a function $f: \mathbb{N} \rightarrow \mathbb{C}$ satisfies (1.3) and for each $\delta>0$ there exists $M=M(\delta)$ such that

$$
\sup _{r \in \mathbb{S}} \sup \{|f(r x)-f(x)-f(r)|: x \in \mathbb{N},(r, x)=1, \omega(x) \geq M(\delta)\}<\delta .
$$


Then there exists an arithmetic additive function $\widetilde{f}: \mathbb{N} \rightarrow \mathbb{C}$ such that

$$
|f(x)-\tilde{f}(x)| \leq 3 \varepsilon \quad \text { for } x \in \mathbb{N} .
$$

Proof. Define a function $g: \mathbb{N} \rightarrow \mathbb{C}$ as follows: $g(1):=f(1)$ and if $x \in \mathbb{N}$ has a canonical factorization $x=r_{1} \cdots r_{s}$ with $r_{1}, \ldots, r_{s} \in \mathbb{S}$ pairwise relatively prime, then we put $g(x):=f(x)-\left(f\left(r_{1}\right)+\cdots+f\left(r_{s}\right)\right)$. Observe that

$$
\left.g\right|_{\mathbb{S}}=0
$$

and $g$ is of the form $f-\widetilde{f}$, where $\tilde{f}$ stands for the unique arithmetic additive function whose values on $\mathbb{S}$ coincide with those of $f$. If we show that $\|g\|_{\text {sup }}$ $\leq 3 \varepsilon$, we will be done.

Fix an arbitrary infinite sequence $R=\left(r_{1}, r_{2}, \ldots\right)$ of pairwise relatively prime elements of $\mathbb{S}$. Set $R_{m}:=\left\{r_{1}, \ldots, r_{m}\right\}$ for $m \in \mathbb{N}$. If $n \in \mathbb{N}$ and $n \leq m$, then we write $R_{m}^{n}$ for the set of all ordered $n$-element sequences of elements from $R_{m}$. Obviously

$$
\operatorname{card} R_{m}^{n}=\frac{m !}{(m-n) !} \quad \text { for } m, n \in \mathbb{N}, n \leq m .
$$

We put

$$
M_{m}(n):=\frac{1}{\operatorname{card} R_{m}^{n}} \sum_{A \in R_{m}^{n}} g\left(\prod_{a \in A} a\right) \quad \text { for } m, n \in \mathbb{N}, n \leq m .
$$

Observe that the decomposition $g=f-\widetilde{f}$, jointly with arithmetic additivity of $\widetilde{f}$ and assumption (1.3), imply that (1.3) remains true after replacing $f$ by $g$. A simple induction and equality (2.9) yield

$$
|g(x)| \leq(\omega(x)-1) \varepsilon \quad \text { for } x \in \mathbb{N}, x \geq 2 .
$$

Evidently, the arithmetic means given by (2.10) satisfy similar inequalities:

$$
\left|M_{m}(n)\right| \leq(n-1) \varepsilon \quad \text { for } m, n \in \mathbb{N}, n \leq m .
$$

This means that for every $n \in \mathbb{N}$ the sequence $\left(M_{m}(n)\right)_{m=n}^{\infty}$ is in $l^{\infty}(\mathbb{C})$. Consider the Banach limit

$$
M(n):=\operatorname{LIM}_{m \rightarrow \infty} M_{m}(n) \quad \text { for } n \in \mathbb{N} .
$$

Some tedious calculations show that

$$
\begin{aligned}
\mid \frac{(m-s-t) !}{m !} \sum_{A \in R_{m}^{s+t}} g\left(\prod_{a \in A} a\right)- & \frac{(m-s) !}{m !} \sum_{B \in R_{m}^{s}} g\left(\prod_{b \in B} b\right) \\
& -\frac{(m-t) !}{m !} \sum_{C \in R_{m}^{t}} g\left(\prod_{c \in C} c\right) \mid \leq \varepsilon
\end{aligned}
$$

for all $s, t \in \mathbb{N}$ and $m \in \mathbb{N}$ such that $s+t \leq m$. Roughly speaking, this follows from the fact that the above difference, after multiplying it by $m$ !, 
may be partitioned into a sum of exactly $m$ ! differences of the form

$$
g\left(\prod_{b \in B} b \prod_{c \in C} c\right)-g\left(\prod_{b \in B} b\right)-g\left(\prod_{c \in C} c\right)
$$

with $B, C$ disjoint. According to condition (1.3) for the function $g$, the absolute values of all such differences are estimated by $\varepsilon$.

The partition process may be described by the transformation $T: R_{m}^{s+t}$ $\rightarrow R_{m}^{s} \times R_{m}^{t}$ given by

$$
R_{m}^{s+t} \ni\left(a_{1}, \ldots, a_{s}, b_{1}, \ldots, b_{t}\right) \mapsto\left(\left(a_{1}, \ldots, a_{s}\right),\left(b_{1}, \ldots, b_{t}\right)\right) \in R_{m}^{s} \times R_{m}^{t} .
$$

Fix $B \in R_{m}^{s}$. When $A$ runs through the domain $R_{m}^{s+t}$ of $T$, the set $B$ appears exactly $\left(\begin{array}{c}m-s \\ t\end{array}\right) t$ ! times as the first element of the pair $T(A)$. Since every such $A$ corresponds to the value $g\left(\prod_{a \in A} a\right)$ with the coefficient $(m-s-t)$ !, the coefficient of $g\left(\prod_{b \in B} b\right)$ should be equal to

$$
\left(\begin{array}{c}
m-s \\
t
\end{array}\right) t !(m-s-t) !=(m-s) !
$$

and, fortunately, it is precisely this.

Therefore, inequality (2.11) gives

$$
\left|M_{m}(s+t)-M_{m}(s)-M_{m}(t)\right| \leq \varepsilon \quad \text { for } m, s, t \in \mathbb{N}, s+t \leq m .
$$

Passing to the Banach limit, applying its linearity and the fact that $\|\mathrm{LIM}\|=1$, we obtain the ordinary $\varepsilon$-additivity of the function $M: \mathbb{N} \rightarrow \mathbb{R}$, i.e.

$$
|M(s+t)-M(s)-M(t)| \leq \varepsilon \quad \text { for } s, t \in \mathbb{N} .
$$

By the classical Hyers theorem [8], there exists an additive function (from the $\operatorname{semigroup}(\mathbb{N},+)$ into $(\mathbb{C},+))$ approximating $M$ to within $\varepsilon$. Such a function must be of the form $s \mapsto c_{R} s$ for some $c_{R} \in \mathbb{C}$ depending only on the fixed sequence $R$. In other words,

$$
\left|M(s)-c_{R} s\right| \leq \varepsilon \quad \text { for } s \in \mathbb{N} \text {. }
$$

This yields

$$
c_{R}=\lim _{n \rightarrow \infty} \frac{M(n)}{n} .
$$

Now we are going to prove that $c_{R}=0$. To this end, define a function $\varphi: \mathbb{N} \rightarrow[0, \varepsilon]$ as follows:

$$
\varphi(k):=\sup \{|g(r x)-g(x)-g(r)|: r \in \mathbb{S}, x \in \mathbb{N}, \omega(x)=k,(r, x)=1\} .
$$

Assumption (2.8), which remains true after replacing $f$ by $g$, guarantees that

$$
\lim _{k \rightarrow \infty} \varphi(k)=0 \text {. }
$$


Fix $n \in \mathbb{N}$ and $y \in \mathbb{N}$ such that $\omega(y)=n$ and let $y=r_{1} \cdots r_{n}$ be the canonical factorization. We have

$$
\begin{aligned}
|g(y)|= & \mid g\left(r_{1} \cdots r_{n}\right)-g\left(r_{2} \cdots r_{n}\right)-g\left(r_{1}\right) \\
& +g\left(r_{2} \cdots r_{n}\right)-g\left(r_{3} \cdots r_{n}\right)-g\left(r_{2}\right)+\cdots \\
& +g\left(r_{n-1} r_{n}\right)-g\left(r_{n}\right)-g\left(r_{n-1}\right) \mid
\end{aligned}
$$

(recall (2.9)). Hence,

$$
\begin{aligned}
|g(y)| & \leq \sum_{i=1}^{n-1}\left|g\left(r_{i} \cdots r_{n}\right)-g\left(r_{i+1} \cdots r_{n}\right)-g\left(r_{i}\right)\right| \\
& \leq \sum_{i=1}^{n-1} \varphi(n-i)=\sum_{i=1}^{n-1} \varphi(i) .
\end{aligned}
$$

Thus,

$$
\frac{|g(y)|}{n-1} \leq \frac{1}{n-1} \sum_{i=1}^{n-1} \varphi(i),
$$

and the arbitrariness of $y$ such that $\omega(y)=n$ allows one to deduce, directly from the definition of $M(n)$, that

$$
\frac{|M(n)|}{n} \leq \frac{1}{n-1} \sum_{i=1}^{n-1} \varphi(i) \underset{n \rightarrow \infty}{\longrightarrow} 0 .
$$

Comparing this with (2.13), we get the desired equality $c_{R}=0$. Furthermore, coming back to (2.12), we deduce that

$$
|M(n)| \leq \varepsilon \quad \text { for } n \in \mathbb{N},
$$

independently of the sequence $R$.

Now, we apply the quoted theorem of Ramsey. Fix $\eta>0$ and consider the following partition of the family $R[n]$ of all $n$-element subsets of $R$ :

$$
\begin{aligned}
R[n]= & \bigcup_{j=0}^{N-1}\left\{A \in R[n]: g\left(\prod_{a \in A} a\right)=r e^{i \vartheta}, \text { where } r \geq \varepsilon+\eta, \vartheta \in\left[\alpha_{j}, \alpha_{j+1}\right]\right\} \\
& \cup\left\{A \in R[n]:\left|g\left(\prod_{a \in A} a\right)\right|<\varepsilon+\eta\right\},
\end{aligned}
$$

where $N$ and $0=\alpha_{0}<\alpha_{1}<\cdots<\alpha_{N}=2 \pi$ are chosen to satisfy

$$
\operatorname{conv}\left\{r e^{i \vartheta}: r \geq \varepsilon+\eta, \vartheta \in\left[\alpha_{j}, \alpha_{j+1}\right]\right\} \cap \bar{B}(0, \varepsilon)=\emptyset \quad \text { for } j=0, \ldots, N \text {. }
$$

By the Ramsey theorem, there exists an infinite subset $Q$ of $R$ such that all $n$-element subsets of $Q$ belong to one of the five parts of the partition. Because of the arbitrariness of the sequence according to which $M(n)$ is calculated, this must be the part $\left\{A \in R[n]:\left|g\left(\prod_{a \in A} a\right)\right|<\varepsilon+\eta\right\}$. If not, the limit $M(n)$ calculated with respect to the sequence $Q$ would lie outside 
the ball $\bar{B}(0, \varepsilon)$, contradicting $(2.15)$. Summarizing this part of the proof, we state the following.

(*) For an arbitrary sequence $R$ of infinitely many relatively prime elements of $\mathbb{S}$, and for any $\eta>0, n \in \mathbb{N}$, there exists an infinite subset $Q \subset R$ such that for all $A \in Q[n]$ we have

$$
\left|g\left(\prod_{a \in A} a\right)\right|<\varepsilon+\eta \text {. }
$$

To finish the proof, fix $x \in \mathbb{N}$ with factorization $x=r_{1} \cdots r_{s}$ and $\eta>0$. By (2.14), there exists $k \in \mathbb{N}$ such that $\varphi(l)<\eta$ provided $l \geq k$. Statement (*) ensures the existence of $y \in \mathbb{N}$ with $(x, y)=1, \omega(y)>k$ and

$$
|g(y)|<\varepsilon+\eta \text {. }
$$

We have $\left|g\left(r_{1} y\right)-g(y)\right|<\eta$, hence $\left|g\left(r_{1} y\right)\right|<\varepsilon+2 \eta$. Since $\mid g\left(r_{1} r_{2} y\right)-$ $g\left(r_{1} y\right) \mid<\eta$, we obtain $\left|g\left(r_{1} r_{2} y\right)\right|<\varepsilon+3 \eta$. Repeating this argument, we arrive at

$$
\left|g\left(r_{1} \cdots r_{s} y\right)\right|<\varepsilon+(s+1) \eta
$$

whereas

$$
\left|g\left(r_{1} \cdots r_{s} y\right)-g(y)-g(x)\right| \leq \varepsilon .
$$

The last two inequalities, jointly with (2.16), yield $|g(x)| \leq 3 \varepsilon+(s+2) \eta$, which ends the proof.

Since the arithmetic additive function $\tilde{f}$ constructed in the above proof coincides with $f$ on $\mathbb{S}$, it is a real function whenever $f$ is real.

3. Stability type result for arithmetic multiplicative functions. Theorems 1 and 2 may be translated to respective stability results for the equation of arithmetic multiplicative functions which do not vanish at any point. We consider the following assumption:

$$
x, y \in \mathbb{N},(x, y)=1 \Rightarrow\left|\frac{f(x y)}{f(x) f(y)}-1\right| \leq \varepsilon .
$$

This is a point of view different from that in [10] and may be regarded as more natural. An assumption of this type was proposed by R. Ger [4] for ordinary exponential mappings. R. Ger and P. Šemrl [5] solved such a stability problem for exponential mappings making use of the Hyers theorem (and some of its variations). Their ideas may be adapted to deduce from Theorem 2 the following one.

ThEOREM 3. Assume that a function $f: \mathbb{N} \rightarrow \mathbb{C} \backslash\{0\}$ satisfies (3.1) for some $\varepsilon \in[0,1)$, and for each $\delta>0$ there exists $M=M(\delta)$ such that

$$
\sup _{r \in \mathbb{S}} \sup \left\{\left|\frac{f(r x)}{f(x) f(r)}-1\right|: x \in \mathbb{N},(r, x)=1, \omega(x) \geq M(\delta)\right\}<\delta .
$$


Then there exists an arithmetic multiplicative function $\tilde{f}: \mathbb{N} \rightarrow \mathbb{C} \backslash\{0\}$ such that

$$
\left|\frac{f(x)}{\widetilde{f}(x)}-1\right| \leq \delta(\varepsilon) \quad \text { and } \quad\left|\frac{\widetilde{f}(x)}{f(x)}-1\right| \leq \delta(\varepsilon) \quad \text { for } x \in \mathbb{N},
$$

where $\delta(\varepsilon)$ is a nonnegative number depending only on $\varepsilon$. Moreover, $\delta(\varepsilon) \rightarrow 0$ as $\varepsilon \rightarrow 0$.

Let us start with a proposition whose proof we only sketch because it is based on the proofs of Theorem 2.1 and Theorem 2.2 from [5].

Proposition. Let $\varepsilon \in[0,1)$. Assume that a mapping $\alpha: \mathbb{N} \rightarrow \mathbb{R}$ satisfies the conditional congruence

$(x, y \in \mathbb{N},(x, y)=1) \Rightarrow \alpha(x y)-\alpha(x)-\alpha(y) \in 2 \pi \mathbb{Z}+[-\arcsin \varepsilon, \arcsin \varepsilon]$, and the following condition: for each $\delta>0$ there exists $M=M(\delta)$ such that

$$
\begin{aligned}
\sup _{r \in \mathbb{S}} \sup \{\operatorname{dist}(\alpha(r x)-\alpha(x)-\alpha(r), 2 \pi \mathbb{Z}) & : x \in \mathbb{N}, \\
(r, x) & =1, \omega(x) \geq M(\delta)\}<\delta .
\end{aligned}
$$

Then there exists a function $p: \mathbb{N} \rightarrow \mathbb{R}$ such that

$$
(x, y \in \mathbb{N},(x, y)=1) \Rightarrow p(x y)-p(x)-p(y) \in 2 \pi \mathbb{Z}
$$

and $|\alpha(x)-p(x)| \leq 3 \arcsin \varepsilon$ for all $x \in \mathbb{N}$.

Proof. Define $I:=[-\arcsin \varepsilon, \arcsin \varepsilon]$ and $\mathcal{P}:=\{(x, y) \in \mathbb{N} \times \mathbb{N}:$ $(x, y)=1\}$. There exist functions $\psi: \mathcal{P} \rightarrow 2 \pi \mathbb{Z}$ and $\varphi: \mathcal{P} \rightarrow I$ such that

$$
\alpha(x y)-\alpha(x)-\alpha(y)=\psi(x, y)+\varphi(x, y) \quad \text { for }(x, y) \in \mathcal{P} .
$$

One may verify that $\psi$ satisfies the following two equations:

$$
\begin{gathered}
\psi(x, y)=\psi(y, x) \quad \text { for }(x, y) \in \mathcal{P} \\
\psi(x, y z)+\psi(y, z)=\psi(x y, z)+\psi(x, y) \quad \text { for }(x, y),(y, z),(z, x) \in \mathcal{P} .
\end{gathered}
$$

Observe now that the following modification of the Hosszú theorem [7] holds: if $\psi$ satisfies (3.5) and (3.6), then there exists a function $\beta: \mathbb{N} \rightarrow \mathbb{R}$ such that

$$
2 \pi \mathbb{Z} \ni \psi(x, y)=\beta(x y)-\beta(x)-\beta(y) \quad \text { for }(x, y) \in \mathcal{P} .
$$

Let $\gamma:=\alpha-\beta$. Then

$$
I \ni \varphi(x, y)=\gamma(x y)-\gamma(x)-\gamma(y) \quad \text { for }(x, y) \in \mathcal{P} .
$$

Condition (3.7) implies that for all $r \in \mathbb{S}$ and $x \in \mathbb{N}$ with $(r, x)=1$ we have

$$
\operatorname{dist}(\alpha(r x)-\alpha(x)-\alpha(r), 2 \pi \mathbb{Z})=|\gamma(r x)-\gamma(x)-\gamma(r)| .
$$

Consequently, in view of assumption (3.4), condition (3.8) and Theorem 2, there is an arithmetic additive function $\delta: \mathbb{N} \rightarrow \mathbb{R}$ such that $|\gamma(x)-\delta(x)| \leq$ $3 \arcsin \varepsilon$ for $x \in \mathbb{N}$. It remains to define $p:=\delta+\beta$. 
Proof of Theorem 3. Write $f(x)=|f(x)| \exp (i \arg f(x))$, where $-\pi<$ $\arg f(x) \leq \pi$. We check that the function $x \mapsto \log |f(x)|$ satisfies the assumptions of Theorem 2 with the constant $-\log (1-\varepsilon)$ instead of $\varepsilon$ and the function $x \mapsto \alpha(x):=\arg f(x)$ satisfies the assumptions of our Proposition (here we need condition (3.2)). Consequently, there exist: a real arithmetic additive function $g$ such that $|g(x)-\log | f(x)|| \leq-3 \log (1-\varepsilon)$ for $x \in \mathbb{N}$ and a real function $p$ which is arithmetic additive modulo $2 \pi \mathbb{Z}$ and such that $|p(x)-\arg f(x)| \leq 3 \arcsin \varepsilon$ for $x \in \mathbb{N}$.

We define a function $\tilde{f}: \mathbb{N} \rightarrow \mathbb{C} \backslash\{0\}$ by the formula

$$
\widetilde{f}(x):=\exp (g(x)+i p(x))
$$

and verify that inequalities (3.3) hold true with

$$
\delta(\varepsilon)= \begin{cases}(1-\varepsilon)^{-3} \sqrt{A(\varepsilon)^{2}+B(\varepsilon)^{2}} & \text { if } \varepsilon<\sqrt{3} / 2 \\ 1+(1-\varepsilon)^{-3} & \text { if } \varepsilon \geq \sqrt{3} / 2\end{cases}
$$

where $A(\varepsilon)=\left(\sqrt{1-\varepsilon^{2}}\right)^{3}-3 \varepsilon^{2} \sqrt{1-\varepsilon^{2}}-(1-\varepsilon)^{3}$ and $B(\varepsilon)=3 \varepsilon-4 \varepsilon^{3}$.

In a similar manner we deduce from Theorem 1 the following one.

ThEOREM 4. Assume that a function $f: \mathbb{N} \rightarrow \mathbb{R} \backslash\{0\}$ satisfies (3.1) for some $\varepsilon \in[0,1)$, and

$$
\liminf _{x \rightarrow \infty} \frac{f(x+1)}{f(x)} \geq 1 .
$$

Then there exists $c \in \mathbb{R}$ such that

$$
\left|\frac{f(x)}{x^{c}}-1\right| \leq \frac{\varepsilon}{1-\varepsilon} \quad \text { and } \quad\left|\frac{x^{c}}{f(x)}-1\right| \leq \frac{\varepsilon}{1-\varepsilon} \quad \text { for } x \in \mathbb{N} \text {. }
$$

\section{References}

[1] P. Erdôs, On the distribution function of additive functions, Ann. of Math. 47 (1946), $1-20$.

[2] Z. Gajda, Invariant Means and Representations of Semigroups in the Theory of Functional Equations, Uniwersytet Śląski, Katowice, 1992.

[3] R. Ger, Almost approximately additive mappings, in: Int. Ser. Numer. Math. 64, Birkhäuser, Basel, 1983, 263-276.

[4] - Superstability is not natural, Rocznik Naukowo-Dydaktyczny WSP w Krakowie, Prace Mat. 159 (1993), 109-123.

[5] R. Ger and P. Šemrl, The stability of the exponential equation, Proc. Amer. Math. Soc. 124 (1996), 779-787.

[6] R. Ger and J. Sikorska, Stability of the orthogonal additivity, Bull. Polish Acad. Sci. Math. 43 (1995), 143-151.

[7] M. Hosszú, On the functional equation $F(x+y, z)+F(x, y)=F(x, y+z)+F(y, z)$, Period. Math. Hungar. 1 (1971), 213-216.

[8] D. H. Hyers, On the stability of the linear functional equation, Proc. Nat. Acad. Sci. USA 27 (1941), 222-224. 
[9] I. Kátai, A remark on additive arithmetical functions, Ann. Univ. Sci. Budapest. Eötvös Sec. Math. 10 (1967), 81-83.

[10] T. Kochanek and M. Lewicki, Stability problem for number-theoretically multiplicative functions, Proc. Amer. Math. Soc. 135 (2007), 2591-2597.

[11] A. Máté, A new proof of a theorem of P. Erdôs, ibid. 18 (1967), 159-162.

[12] A. Rényi, On a theorem of P. Erdôs and its application to information theory, Mathematica 1 (1959), 341-344.

[13] S. M. Ulam, Problems in Modern Mathematics, Wiley, New York, 1960.

[14] E. Wirsing, A characterization of $\log n$ as an additive arithmetic function, in: Sympos. Math. IV, Academic Press, London, 1970, 45-57.

Institute of Mathematics

Silesian University

Bankowa 14

40-007 Katowice, Poland

E-mail: t_kochanek@wp.pl

Received on 2.4.2007

and in revised form on 9.2.2008 